\section{e0389 CLINICAL PROFILE OF PREMENOPAUSAL WOMEN WITH CORONARY HEART DISEASE}

doi:10.1136/hrt.2010.208967.389

RongJing DING, Dayi Hu. Heart and Vascular Center, Beijing University People's hospital, Beijing China

Objective To study the clinical profile of premenopausal women with coronary heart disease.

Methods 116 premenopausal women with chest pain were classified into coronary heart disease group and control group by coronary angiography. Risk factors, clinical symptoms and the coronary angiographic characteristics were investigated retrospectively.

Results The risk factors of premenopausal women with coronary heart disease were hypertension, diabetes and hyperlipidaemia. Typical angina pectoris was an important character. The typical change of ECG in premenopausal women with coronary heart disease was elevation or depression of ST, but not T wave. The sensitivity and specificity of Exercise stress testing or SPECT for premenopausal women with coronary heart disease were $67.7 \%$ and $32.2 \%, 40.9 \%$ and $59 \%$, respectively. Single vessel coronary lesion was found more frequently in Premenopausal Women with coronary heart disease, and the left anterior disending artery was the most frequently involved vessel.

Conclusion Hypertension, Diabetes and/or hyperlipidaemia are major risk factors in premenopausal women with coronary heart disease. Women with typical angina pectoris and ST changes should be cautioned coronary heart disease. Nonvasive testing is a poor diagnosis method for young women with coronary heart disease, but can be used as exclusive marker.

\section{e0390 RELATIONSHIP OF NO OR SLOW REFLOW OF PATIENTS WITH ACUTE MYOCARDIAL INFARCTION AFTER PCI WITH THE STRESSING BLOOD SUGAR AND HS-CRP}

doi:10.1136/hrt.2010.208967.390

${ }^{1}$ Wei Peng, ${ }^{1}$ Fu Oiang, ${ }^{2}$ Bai Jie, ${ }^{1}$ Sheng Yang, ${ }^{1}$ Liu Yi, ${ }^{1}$ Lu Yi. ${ }^{1}$ Department of Cardiology, Xuzhou Central Hospital; ${ }^{2}$ Changhai Hospital of Shanghai

Objective To study releation between the stressing blood sugar and hs-CRP and the no or slow reflow occurred in patients with acute myocardial infarction after primary PCI.

Methods 253 patients be suspected STEMI with chest pain were admitted to this study, all patients were selected from heart center of our hospital and the time of D-to-B was less than $6 \mathrm{~h}$. were divided into group of no or slow reflow and group of normal control according to the results of CAG.

Results 26 patients had no or slow reflow, and the stressing blood sugar, hs-CRP of group with no reflow were super to the control group obviously $(\mathrm{p}<0.05$ or $\mathrm{p}<0.01)$.

Conclusion detection of stressing blood sugar and hs-CRP were correlated to the occuration of no or slow reflow after primary PCI, and can be used to predict the Prognosis.

\section{e0391 VALUE OF TESTING OF CAROTID ARTERY PLAQUE JOINT HS-CRP TO THE DEGREE OF CORONARY STENOSIS IN PATIENTS WITH CORONARY HEART DISEASE}

doi:10.1136/hrt.2010.208967.391

Sheng Yang, Fu Qiang, Wei Peng, Li Zhenyong Yi. Department of Cardiology, Xuzhou Central Hospital

Objective To study the predictive value of score of intima-media thickness (IMT), Crouse in carotid artery plaque, and serum levels of high sensitivity C-reactive protein (hs-CRP) in the degree of coronary stenosis of patients with coronary artery disease.

Methods A total of 110 patients suspected coronary heart disease with chest pain were admitted to this study. They were divided into two groups: group of coronary heart disease and group of normal control according to the results of CAG. All patients underwent carotid ultrasonography within a week, and carried out the testing of serum concentrations of hs-CRP in second hospitalised day, to assess the determining and forecasting value of score of IMT and Crouse in carotid artery plaque, combining with serum levels of hs-CRP in the degree of coronary stenosis of patients with coronary artery disease.

Results Serum concentrations of hs-CRP, score of IMT, Crouse of group CHD were significantly higher than those of group non-CHD $(p<0.01)$, and each mentioned indicators in group CHD was positively correlated with the degree of coronary stenosis $(r>0.6$, $\mathrm{p}<0.05)$. Taking Crouse points $\geq 10.7$ as a standard, its sensitivity was $78.5 \%$, positive predictive value was $89.9 \%$, specificity was $79.0 \%$, negative predictive value was $58.0 \%$.

Conclusion There was a high consistency between serum concentrations of hs-CRP, score of IMT, Crouse and the degree of coronary stenosis, so we can use serum concentrations of hs-CRP, score of IMT, Crouse as a prediction method to coronary heart disease in patients with carotid artery atherosclerosis.

\section{e0392 RELATIONSHIP BETWEEN RETINAL VASCULOPATHY AND CORONARY ARTERY DISEASE}

doi:10.1136/hrt.2010.208967.392

${ }^{1}$ Ren Wenlin, ${ }^{1}$ Chen Jun, ${ }^{2}$ Xu Oing, ${ }^{1}$ Li Guihua, ${ }^{2}$ Zhang Wanping, ${ }^{2}$ Wu Baojian, ${ }^{1}$ Pi Lin, ${ }^{1}$ Peng Fusong. ${ }^{1}$ Cardiac Center, Beijing Chuiyangliu Hospital, Beijing, China; ${ }^{2}$ Ophthalmology Department, Beijing Chuivangliu Hospital, Beïing, China

Background and objective Studies showed that atherosclerosis is a systemic disease. Parameters representing peripheral artery atherosclerosis, such as decreased ankle-brachial index (ABI), and increased carotid artery intima-media thickness (CIMT), are well correlated with coronary artery disease. However, these are indirect indicators provided by ultrasound examination. Eyes are good windows, through which we can observe vascular anatomy and function in vivo directly and clearly. Our study was to explore the correlation of retinal vascular diameter and arteriole-to-venule ratio (AVR) on the retinal photographs to extent and severity of coronary artery disease $(\mathrm{CAD})$ angiographically.

Methods From January 2007 to February 2008, the patients admitted in CCU and Department of Cardiovascular Disease of Beijing Chuiyangliu Hospital with diagnosed or suspected of CAD were selected to accept coronary artery angiography using standard Judikin's technique. According to Gensini score, the degree and extent of coronary atherosclerosis were visually evaluated and scored by 2 expert cardiologists. The calibres of individual retinal arteriole and venule coursing through a zone located at 1 to 1.5 disc diameter from the optic disc margin were measured on the digital retinal photographs using a computer-assisted method by two trained oculists who had no knowledge of the patients' condition of coronary artery angiogram, and the arteriole-to-venule ratio was calculated.

Results 1.114 patients were enrolled, including 61 men and 53 women, aged from 34 to 82 years $(60.16 \pm 10.34 \mathrm{y})$. The diagnosis of $\mathrm{CAD}$ was confirmed in 85 patients (CAD group) and the other 29 patients with negative results (control group) angiographically. There were no significant differences between two groups in terms of baseline clinical characteristics. 2. The result of coronary artery angiography: 85 patients $(74.57 \%)$ were diagnosed as CAD, and the other 29 patients $(25.43 \%$ ) were excluded from $\mathrm{CAD}$. In CAD group, 
the severity of the coronary lesions was assessed by Gensini scoring system, a method that assigns a different severity score depending on the degree of luminal narrowing and the geographical importance of their locations. 25 patients scored $-40,26$ patients scored 41-80, 23 cases scored $81-120$, and 11 cases scored greater than 120.3 . The results of the retinal vascular measurement and calculation: The mean retinal arteriole diameter (upper temporal branch and inferior temporal branch) in CAD group was significantly smaller than that in control group $(p<0.05)$. The mean retinal venule diameter (upper temporal branch and inferior temporal branch) in CAD group was significantly greater than that in control group $(p<0.05)$. The mean retinal AVR (both in upper temporal branch and inferior temporal branch) in $\mathrm{CAD}$ group was much smaller than that in control group $(p<0.05)$. 4. The correlation analysis between retinal vascular diameter, AVR and the severity of coronary atherosclerosis: the numerical values of retinal artery diameter and AVR in patients with Gensini score group $81-120$ and $>120$ were significantly smaller than that with Gensini score $\leqq 40(p<0.05)$, but the calibre of retinal venule in patients with Gensini score group 81-120 and $>120$ was significantly greater than that with Gensini score $\leqq 40$ $(p<0.05)$, there were no significant differences between the other groups. With Pearson correlation analysis to analyse the correlation of retinal AVR to Gensini score, the result showed that in CAD patients, the retinal AVR was negatively correlated to the Gensini score $(p<0.01)$, With partial correlation analysis and controlling of other influencing factors, such as hypertension and diabetes, the negtive correlation didn't change.

Conclusion In our study, the retinal vascular diameter and AVR are well correlated to the severity of coronary artery disease. In CAD patients, the retinal arteriole calibre and AVR are significantly negatively correlated to Gensini score, and the retinal venule diameter is strongly positively correlated to Gensini score.

\section{e0393 SIGNIFICANCE OF OXIDISED LOW-DENSITY LIPOPROTEININ CORONARY ATHEROSCLEROTIC HEART DISEASE}

doi:10.1136/hrt.2010.208967.393

Xiaoping Meng, Chang Li. Jilin University No 2 Hospital

Objective In this study, control group are people with normal coronary arteries. This study is to discuss the relationship between $\mathrm{LDL}, \mathrm{OX}-\mathrm{LDL}$ and control group, SAP group, UAP group, AMI guoup and contaction of LDL and OX-LDL.

Methods Experimental group are $300 \mathrm{CHD}$ patients without taking lipid-lowering drugs in one month who admittde in the depatrment of cardiolysis from August 2008 to August 2009. They are divided into SAP group (100 cases), UAP group (100 cases), AMI (100 cases). The control group are 100 cases of patients who are randomly selected and confirmed without CHD by coronary angiography in the same period in our department. Information includs gender, age, smoking, drinking, hypertension, diabetes, levels of LDL and OX-LDL. We analysis patients' basic information, the level and correlation of LDL and OX-LDL in control group, SAP group, UAP group and AMI group.

Results 1 . There are no significant differents between control group and CHD groups in basic information ( $p>0.05)$. 2. Concentration of $\mathrm{LDL}$ in CHD group is significant difference compared with control group $(p<0.01)$. Concentration of LDL in UAP group is no significant difference compared with SAP group ( $p>0.05)$. Concentration of LDL in AMI group is significant difference compared with SAP group $(p<0.01)$. Concentration of LDL in AMI group is no significant difference compared with UAP group $(p>0.05)$. 3. Concentration of OX-LDL in CHD group is significant difference compared with control group $(p<0.01)$. Concentration of OX-LDL in UAP group is significant difference compared with SAP group $(p<0.05)$.
Concentration of OX-LDL in AMI group is significant difference compared with SAP group $(p<0.01)$. Concentration of OX-LDL in AMI group is significant difference compared with UAP group $(p<0.01)$. 4. There is no correlation between concentration of LDL and OX-LDL in all groups ( $\mathrm{p}>0.05)$.

Conclusions 1 . We confirm that $\mathrm{LDL}$ and $\mathrm{OX}-\mathrm{LDL}$ are risk factors for $\mathrm{CHD}$. There is no significant differences about the concentration of $\mathrm{LDL}$ in CHD groups, however, there is significant differences about the concentration of OX-LDL in CHD groups. The level of OX-LDL is in escalating trend. 2 There is no correlation between concentration of LDL and OX-LDL in all groups $(\mathrm{p}>0.05)$ and OX-LDL play a more important role in the process of CHD. Compared with LDL, mensurating OX-LDL is more meaningful in the treatment and prevention of $\mathrm{CHD}$

\section{e0394 THE RELATIONSHIP BETWEEN OBSTRUCTIVE SLEEP APNEA AND ENDOTHELIN-1 PLASMA LEVELS IN PATIENTS WITH CORONARY HEART DISEASE}

doi:10.1136/hrt.2010.208967.394

Zhao Qing, Liu Zhihong, Zhao Zhihui, Luo Qin, Zhang Hongliang, Wang Yong. Fuwai Hospital, Beijing, China

Objective Obstructive sleep apnea (OSA) is an independent risk factor for cardiovascular morbidity and mortality. The mechanism is unknown, but recent studies provide evidence that endothelial dysfunction might contribute. So we investigate the relationship between obstructive sleep apnea and endothelin-1 (ET-1) plasma levels in patients with coronary heart disease.

Methods 287 patients with coronary heart disease were enrolled and an overnight polysomnography was performed to all of them. According to the apnea-hypopnoea index, the patients were divided into four groups: no OSA group ( $\mathrm{AHI}<5, \mathrm{n}=58$ ), mild OSA group $(5 \leq \mathrm{AHI}<15, \mathrm{n}=82)$, moderate OSA group $(15 \leq \mathrm{AHI}<30, \mathrm{n}=70)$, severe OSA group (AHI $\geq 30, n=77$ ). Plasma levels of endothelin-1 were detected to all the patients.

Results Compared with no and mild OSA group, endothelin-1 plasma levels in severe OSA group elevated significantly $(p=0.009)$, even after analysis in a general linear model with correction for confounders. Plasma ET-1 levels showed an increasing trend within no OSA, mild OSA and moderate OSA group, however no statistically significant was observed between no OSA group and patients with mild or moderate OSA, respectively $(p=0.421, p=0.226)$.

Conclusion Among patients with coronary heart disease, plasma ET-1 levels elevated significantly in severe OSA group. This might support that severe OSA has more effects on endothelium function for coronary heart disease than mild and moderate OSA.

\section{e0395 CORRELATION BETWEEN SERUM LEVELS OF CATHEPSIN S AND SEVERITY OF ATHEROSCLEROSTIC LESIONS OF CORONARY ARTERIES IN CHINESE}

doi:10.1136/hrt.2010.208967.395

Liu Yu-sheng, Hao Lin, Jiang Wei-dong, Lu Qing-hua, Wang Yong- Mei. Department of Cardiology, The Second Hospital of Shandong University, Jinan

Objective Previous studies have showed that cathepsin S(CatS) is upregulated in atherosclerosis lesions in humans. We try to deplore the association between serum levels of CatS and its inhibitor cystatin $\mathrm{C}(\mathrm{Cys} \mathrm{C})$ and the severity of atherosclerostic lesions of coronary arteries in Chinese.

Methods and results 107 coronary atherosclerositic diseases (CAD) and 48 controls were recruited and all subjects consisting were verified by selective angiography, the the severity of lesions of 